\title{
.
}

Navin Dewaji*

\section{HACIENDO ENTREGA DE LA ENERGÍA POSITIVA DE INDIA}

La búsqueda de la autosuficiencia energética en India y las acciones contra el cambio climático fueron iniciadas en el país a finales de la década de 1980. La visión renovable del Gobierno indio, junto con el marco regulatorio, las tarifas preferenciales, los incentivos fiscales y el énfasis en una base de fabricación sólida allanaron el camino para un rápido crecimiento en el sector eólico. Desde principios de la década de 2000, las instalaciones eólicas se han multiplicado en el país, siendo hoy día el cuarto mayor mercado mundial. En los últimos años, el mercado indio se ha enfrentado a problemas a raíz de la transición del régimen de tarifas preferenciales al actual régimen de subastas, dando como resultado una caída en la nueva capacidad instalada. Sin embargo, los fundamentos a largo plazo de la industria continúan siendo sólidos y el panorama en el sector parece prometedor, con un desempeño importante de las fuentes eólicas y solares en la transición energética, aparte de los - aún por explorar en el país - sectores de repotenciación y energía eólica offshore. Siemens Gamesa, líder mundial en el sector, está lista para acelerar el sector eólico indio y acercarlo hacia sus objetivos de energía renovable.

Palabras clave: Siemens Gamesa, India, energías renovables, solar, eólica, perspectivas.

Clasificación JEL: L52, L94, M11, N75, O13, O33, Q42, Q55.

\section{Evolución del sector eólico en India}

El cuarto mayor mercado eólico mundial tiene una larga historia de hitos. La historia del sector eólico indio comienza en la década de 1980, cuando el país se hace consciente de la importancia de las fuentes de energía sostenible y de la autosuficiencia.

En los primeros años de la década de los ochenta, el Gobierno de India se centró en la

\footnotetext{
* CEO de Siemens Gamesa Renewable Power Pvt Ltd. Traducción: Oficina Económica y Comercial de Nueva Delhi. Versión de septiembre de 2020.

DOI: https:/doi.org/10.32796/bice.2020.3128.7092
}

demostración de la tecnología eólica y la evaluación de sus propios activos eólicos. La primera instalación de turbinas eólicas conectadas a la red fue realizada con la participación y ayuda del sector privado y los programas de promoción del Departamento de Fuentes de Energía no Convencionales indio. La participación del sector privado se incrementó de manera significativa tras las políticas de liberalización de la década de 1990, con el establecimiento de instalaciones de producción de equipos por parte de los nuevos fabricantes de equipo original (OEM, por sus siglas en inglés) y la entrada de actores extranjeros. 


\subsection{Marco regulatorio y era de las Feed- in-Tariffs (FiT)}

La creación de la Agencia de Desarrollo de Energías Renovables de India (IREDA, por sus siglas en inglés) en 1982, de un Ministerio de Fuentes de Energía no Convencional en 1992 y la aprobación de la Ley de Electricidad en 2003 proporcionaron un marco legislativo e institucional para promover la energía renovable conectada a la red con tarifas definidas y asistencia financiera/fiscal en el mercado eléctrico.

Los desarrollos comerciales y las adiciones de capacidad de generación de energía eólica aumentaron drásticamente con las políticas gubernamentales de incentivos del sector. Algunas de estas políticas, como las depreciaciones aceleradas en inversiones de capital y las exenciones fiscales sobre el impuesto de electricidad, supusieron unos incentivos que llamaron la atención de actores tanto nacionales como internacionales.

Durante dos décadas, las tarifas preferenciales FiT han estado brindando el apoyo necesario para el desarrollo del sector eólico al atraer a grandes actores privados. Es importante destacar que alrededor del $95 \%$ de las inversiones realizadas en el sector eólico provinieron del sector privado en este periodo. Además, en 2016, como parte de la Convención Marco de las Naciones Unidas sobre el Cambio Climático, el Gobierno de India (en adelante Gdl) adoptó un firme compromiso en la Conferencia sobre el Cambio Climático de $\mathrm{Pa}$ rís (COP21) para lograr el $40 \%$ de la cuota de generación instalada en 2030 a partir de combustibles no fósiles, incluyendo energía eólica, solar y gran hidroeléctrica, junto con otras renovables, brindando así una visión a largo plazo para la industria. El Ministerio de Energías
Nuevas y Renovables (MNRE, por sus siglas en inglés), antiguo Ministerio de Energías no Convencionales, anunció un ambicioso objetivo de 175 GW para 2022 (de los cuales 60 GW son contribución eólica), reiterando sus objetivos de transición energética.

\subsection{Perspectiva del mercado}

Desde el anuncio del objetivo nacional, el sector eólico ha mostrado siempre un alto grado de compromiso por parte de todas las partes interesadas. Los OEM, uno de los principales actores del sector, han realizado inversiones significativas en el país, estableciendo una sólida base de fabricación de $10.000 \mathrm{MW}$. La tecnología eólica ha tenido un desarrollo significativo, desde las anteriores plataformas de menos de $1 \mathrm{MW}$ hasta las actuales de $3 \mathrm{MW}$, que ofrecen una mayor eficiencia. Se estima que para 2022, de cumplirse el objetivo marcado de $175 \mathrm{GW}$, el sector eólico indio pueda llegar a generar 1,3 millones de puestos de trabajo.

La transición hacia la fijación de tarifas y la asignación de capacidad basada en subastas con tarifas insostenibles que han llegado hasta las 2,4 rupias/ $\mathrm{kWh}(0,028$ euros) ha ejercido una enorme presión en toda la cadena de valor, creando una competencia indebida y dificultades financieras para los OEM y los fabricantes de componentes. Adicionalmente, los techos tarifarios en las subastas han mantenido a los desarrolladores escépticos a la hora de participar, resultando en subscripciones insuficientes y retrasos. Si bien las instalaciones acumuladas ascienden a 37,9 GW en julio de 2020 , los problemas relativos a la disponibilidad de terrenos y las limitaciones de la infraestructura de red han afectado de forma $\triangleright$ 
severa a los proyectos de instalación eólica. Las adiciones anuales de capacidad eólica, que alcanzaron su pico en 2017 con cerca de 4,1 GW, cayeron en 2018 a 2,3 GW, manteniéndose estancadas en 2019, con 2,4 GW.

La desaceleración de las instalaciones eólicas en los dos últimos años se ha intensificado durante la primera mitad de 2020 con los confinamientos derivados de la pandemia mundial de la COVID-19. Sin embargo, el Gdl ha tomado diversas medidas para ayudar a minimizar el impacto del confinamiento, permitiendo una extensión en los plazos de entrega de los proyectos abiertos y el movimiento de materiales para proyectos de energía renovables, entre otros.

Según fuentes de investigación de mercado, la instalación anual para el año 2020 se espera que sea del orden de 1,5-2 GW, menor al año anterior. En el corto plazo, se estima una clara reducción de las instalaciones eólicas que se encuentren en el rango de 3,5-4 GW frente a las anteriores proyecciones de 6-7 GW. A pesar de las reducciones de los volúmenes de mercado a corto plazo, India continúa teniendo una fuerte perspectiva, posicionándose entre los tres principales mercados eólicos a nivel mundial, después de China y EE UU.

\section{El viaje de Siemens Gamesa en India}

Siemens Gamesa Energía Renovable es uno de los mayores proveedores de soluciones renovables del mundo. Desde su creación en 2009, la compañía ha llevado a cabo de forma exitosa proyectos por más de 6.900 MW alrededor de toda India. La flota operativa de parques eólicos de Siemens Gamesa se distribuye en ocho estados, con oficinas de ventas y operaciones en seis ubicaciones, cuatro plantas de fabricación, un centro de ingeniería y 38 oficinas distribuidas por todo el país. La compañía emplea actualmente a más de 2.500 personas.

La historia de la compañía en India comenzó con Gamesa Corporación Tecnológica, $\triangleright$

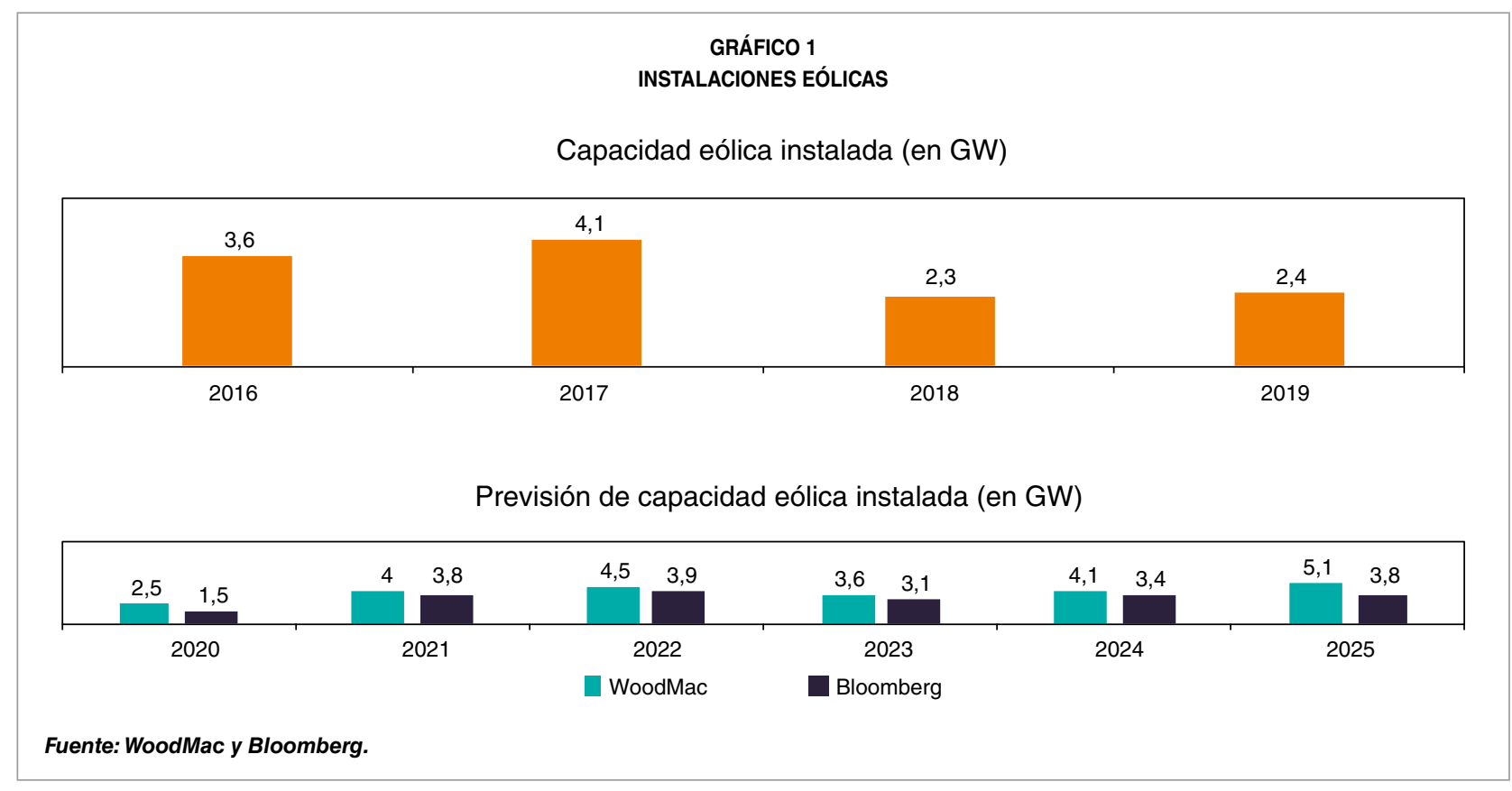


que hizo su incursión en el país en 2009 con su primera planta de fabricación de aerogeneradores de 850 kW en RedHills, Chennai. A los dos años de operaciones en el país, lanzó una turbina de $2 \mathrm{MW}$ con un motor de 97 metros de diámetro para satisfacer la creciente demanda en el mercado eólico. En 2013 inició en Mamandur, Tamil Nadu, una nueva planta de fabricación de nacelles de última generación. En 2015, expandió su cartera de productos con otra turbina eólica de plataforma de $2 \mathrm{MW}$ y un diámetro de motor de 114 metros, y lo amplió a 122 metros en un breve periodo de dos años. En 2016, Siemens Gamesa estableció su centro de fabricación integrado en Nellore, Andhra Pradesh, equipado para manufacturar toda la cadena de valor del negocio eólico y los inversores centrales fotovoltaicos del negocio solar.

La fusión de Gamesa Corporación con Siemens Wind Power en 2016-2017 la ayudó a posicionarse como líder mundial en la industria eólica, al derivar las sinergias de ambos líderes de la industria, proporcionando así un valor añadido a todos los grupos de interés.

En 2018, se estableció un nuevo hub de I+D en Bangalore, conformado por más de quinientos ingenieros talentosos que reiteran el enfoque de la empresa en la tecnología y la innovación.

Siemens Gamesa India entró en el negocio solar en 2015, ofreciendo un amplio espectro de soluciones solares Engineering, Procurement and Construction (EPC). Actualmente, Siemens Gamesa India ha entregado más de $400 \mathrm{MW}$ de proyectos de energía solar.

Siemens Gamesa también ha demostrado la capacidad de los híbridos en India, siendo la primera compañía en entregar un proyecto híbrido solar-eólico comercial. El proyecto, que tendrá una capacidad de 78,8 MW (50 MW de energía eólica y 28,8 MW de solar), será realizado en el estado de Karnataka. La entidad fijó un nuevo punto de referencia en 2019 , al convertirse en la primera compañía energética del mundo en ganar el prestigioso premio Deming por estándares de calidad.

Siemens Gamesa lidera actualmente el camino eólico en el país, lanzando recientemente una nueva generación de turbinas eólicas SG 3.4-145. La turbina ofrece un incremento de generación energética del $48 \%$ y reduce el coste nivelado de la energía en comparación con su predecesor de plataforma de $2 \mathrm{MW}$. La turbina está diseñada para los vientos indios y será fabricada en India. Siemens Gamesa se encuentra actualmente afianzada para reforzar la posición de liderazgo en el mercado y acelerar la lucha por las renovables.

CUADRO 1

SIEMENS GAMESA INDIA

\begin{tabular}{|l|l|}
\hline \multicolumn{2}{|l|}{ Datos clave } \\
\hline Capacidad instalada & $>6.900 \mathrm{MW}$ \\
\hline Cuota de mercado en 2019 & Líder del mercado con un $30 \%$ \\
\hline $\mathrm{N} .^{\circ}$ de empleados & $>2.500$ \\
\hline Presencia industrial & $\begin{array}{l}4 \text { plantas productivas y } 1 \text { centro } \\
\text { de ingeniería }\end{array}$ \\
\hline Fuente: elaboración propia. & \\
\hline
\end{tabular}

\section{Retos y camino a seguir}

El mercado eólico indio se ha ido transformando con el paso de los años en un mercado maduro, competitivo y sensible al precio. Mientras el Gdl ha tomado medidas para abordar los problemas de los techos tarifarios en subastas y ha relajado las condiciones de las licitaciones publicadas recientemente, los retos de infraestructuras de red, disponibilidad de terrenos y de salud financiera de las compañías distribuidoras energéticas (DISCOM) continúan siendo una gran preocupación. Estos desafíos suponen problemas estructurales de $\triangleright$ 
un mercado que necesita políticas adecuadas e intervenciones regulatorias.

A pesar de todos los desafíos de un mercado impulsado por las subastas, el sector eólico continúa siendo una fuente energética competitiva, y seguirá siendo un componente clave para conducir la transición energética india. Si continúa el modelo actual de subastas, se estima un volumen anual instalado de entre 3,5 y $4 \mathrm{GW}$, y este volumen podría aumentar en la medida en que lo haga la disponibilidad de red y tierra. Las tarifas competitivas y los parques eólicos con un bajo factor de carga eólica (PLF, por sus siglas en inglés) requieren que las turbinas eólicas ofrezcan un coste nivelado de energía ( $L C o E$, por sus siglas en inglés) atractivo para que la energía eólica siga siendo competitiva.

Es esencial que las tarifas mejoren desde los actuales niveles de 2,8-2,9 rupias/ $\mathrm{kWh}$ $(0,032 € / \mathrm{kWh})$ a un rango sostenible que respalde la rentabilidad de todas las partes implicadas a lo largo de la cadena de valor. Se necesita un mayor despliegue de tecnologías digitales para el mantenimiento de activos y el cambio a plataformas de turbinas eólicas de mayor calificación, junto con soluciones híbridas basadas en el almacenamiento energético, para atender la dinámica del mercado.

La repotenciación de viejos parques eólicos brindaría una nueva oportunidad para instalar las últimas tecnologías, para obtener un mejor uso de los ricos recursos eólicos de un país cuya tierra es finita. Se estima que cerca de $5.000 \mathrm{MW}$ de capacidad instalada en viejas turbinas pueden ser reemplazados por turbinas de última tecnología. Esto podría proporcionar una mayor generación energética por MW por ubicación. Aunque Siemens Gamesa ha realizado intentos de repotenciación en un par de proyectos, no suscita un elevado interés entre los desarrolladores debido a diversas razones, entre las que se encuentran la tenencia compartida de los terrenos, un gasto de capital (capex) más alto o los problemas de evacuación de energía aparte de la tarifa (antiguas tarifas ofrecidas por las DISCOM durante el periodo del Power Purchase Agreement, PPA). Además, la larga costa del país $(7.500 \mathrm{~km})$, con aguas relativamente poco profundas, debería ser analizada por el enorme potencial eólico offshore que ofrece.

Con sólidos fundamentos y un compromiso a nivel nacional, la industria eólica está destinada a volver a una trayectoria de alto crecimiento. Con la vista puesta en el año 2030, el objetivo masivo marcado por el Gdl, por el que se pretende una instalación renovable de $450 \mathrm{GW}$ (140 GW de viento), sitúa al país en el mapa mundial como uno de los mercados eólicos con mayor potencial, ofreciendo energía limpia y asequible.

\section{Bibliografía}

Chandrasekaran, K. (13 de abril de 2020). India's wind installation for 2020 likely to fall drastically. ETEnergyWorld. https://energy.economictimes. indiatimes.com/news/renewable/indias-windinstallation-for-2020-likely-to-fall-drastically/ 75114659

GWEC (2020). India wind outlook towards 2022: Looking beyond headwinds. https://gwec.net/india-wind-outlook-towards-2022-looking-beyond-headwinds/\#: :text=The $\% 20$ India $\% 20$ Wind\%200utlook\%202020, an\%20assessment\%20on\%20the\%20forecast

\section{Páginas web consultadas}

Bloomberg New Energy Finance. https://about.bnef. $\mathrm{com} /$

CEA: Central Electricity Authority. http://cea.nic.in/

CEEW: Council on Energy, Environment and Water, India. https://www.ceew.in/renewables 
Navin Dewaji

GWEC: Global Wind Energy Council. https://gwec.net/

IREDA: Indian Renewable Energy Development Agency. https://www.ireda.in/

IRENA: International Renewable Energy Agency. https://www.irena.org/
MNRE: Ministry of New and Renewable Energy, India. https://mnre.gov.in

MoP: Ministry of Power, India. https://powermin.gov. in/en

Wood Mackenzie. https://www.woodmac.com/ 